\title{
Perturbing laser field dependent high harmonic phase modulations
}

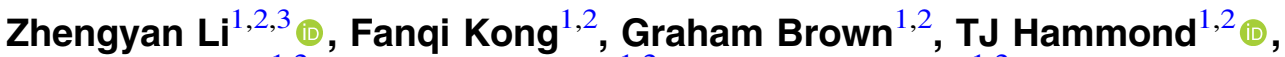 \\ Dong-Hyuk Ko ${ }^{1,2}$, Chunmei Zhang ${ }^{1,2}$ and $\mathbf{P}$ B Corkum ${ }^{1,2}$ \\ ${ }^{1}$ Department of Physics, University of Ottawa, Ottawa, Canada \\ ${ }^{2}$ Joint Attosecond Science Laboratory, National Research Council and University of Ottawa, Canada \\ ${ }^{3}$ School of Optical and Electronic Information, Huazhong University of Science and Technology, Wuhan, \\ China \\ E-mail: Chunmei.Zhang@uottawa.ca and pcorkum@uottawa.ca
}

Received 21 March 2018, revised 14 April 2018

Accepted for publication 1 May 2018

Published 23 May 2018

\begin{abstract}
A perturbing laser pulse modulates and controls the phase of the high harmonic radiation driven by an intense fundamental pulse. Thus, a structured wave front can impress a specific spatial phase onto the generated high harmonic wave front. This modulation procedure leads to alloptical spatial light modulators for VUV or XUV radiation created by high harmonic generation. Here, through theoretical analysis and experiment, we study the correlation between the high harmonic phase modulations and the perturbing laser field amplitude and phase, providing guidelines for practical high harmonic spatial light modulators. In addition, we show that the petahertz optical oscilloscope for measuring electric fields of a perturbing beam is most robust using low order harmonics, far from the cut-off.
\end{abstract}

Keywords: high harmonic generation, wave front control, spatial light modulator

(Some figures may appear in colour only in the online journal)

\section{Introduction}

Spatial light modulators (SLMs) control the transverse distributions of the laser light amplitude and/or phase with programmable computer control [1], so complicated topological structures such as Bessel beams [2] or optical vortices can be generated [3]. With controlled laser beams, SLMs have found applications in optical tweezing and trapping [4, 5], holography [6, 7], and laser processing [8]. In ultrafast optics, temporal pulse shaping for femtosecond lasers is achieved with an SLM, which controls the spectral amplitude and phase of femtosecond pulses [9]. Most commercial SLMs work for visible light.

Our aim is to extend their feasibility to a broader spectral range [10]. We concentrate on extreme ultraviolet (XUV) radiation where high harmonic generation provides an ultrafast, coherent light source for attosecond sciences [11], coherent imaging [12, 13], XUV nonlinear optics [14], and seeding free electron lasers [15]. Most applications require focusing, but due to weak refraction and strong absorption in media as well as the need for high surface quality, XUV optics are challenging to manufacture [16]. Usually, grazing incident toroidal mirrors or multi-layer mirrors focus high harmonic radiation for enhanced XUV photon fluence required by some applications [17-20]. A reflective Fresnel zone plate is another option for focussing narrow linewidth high harmonic beams [21]. In order to reduce the aberration of the high harmonic radiation, either intrinsic to the generation process or induced by the XUV optics, it is important to control the high harmonic wave front.

To reduced aberrations or to produce novel topological spatial structures, high harmonic technology offers a series of control parameters such as the density of the generating medium [22], the astigmatism of the driving laser field [23], or a two-color combination of the driving laser field [24]. In addition, complicated topological structures can be applied to high harmonic spatial profiles by, for example, controlling the driving laser optical angular momentum for high harmonic optical vortices [25-27], or manipulating the laser polarization for XUV vector beams [28]. The non-collinear geometry of two controlled laser beams has realized different 'alloptical' high harmonic optical devices, such as generation of 
(a)
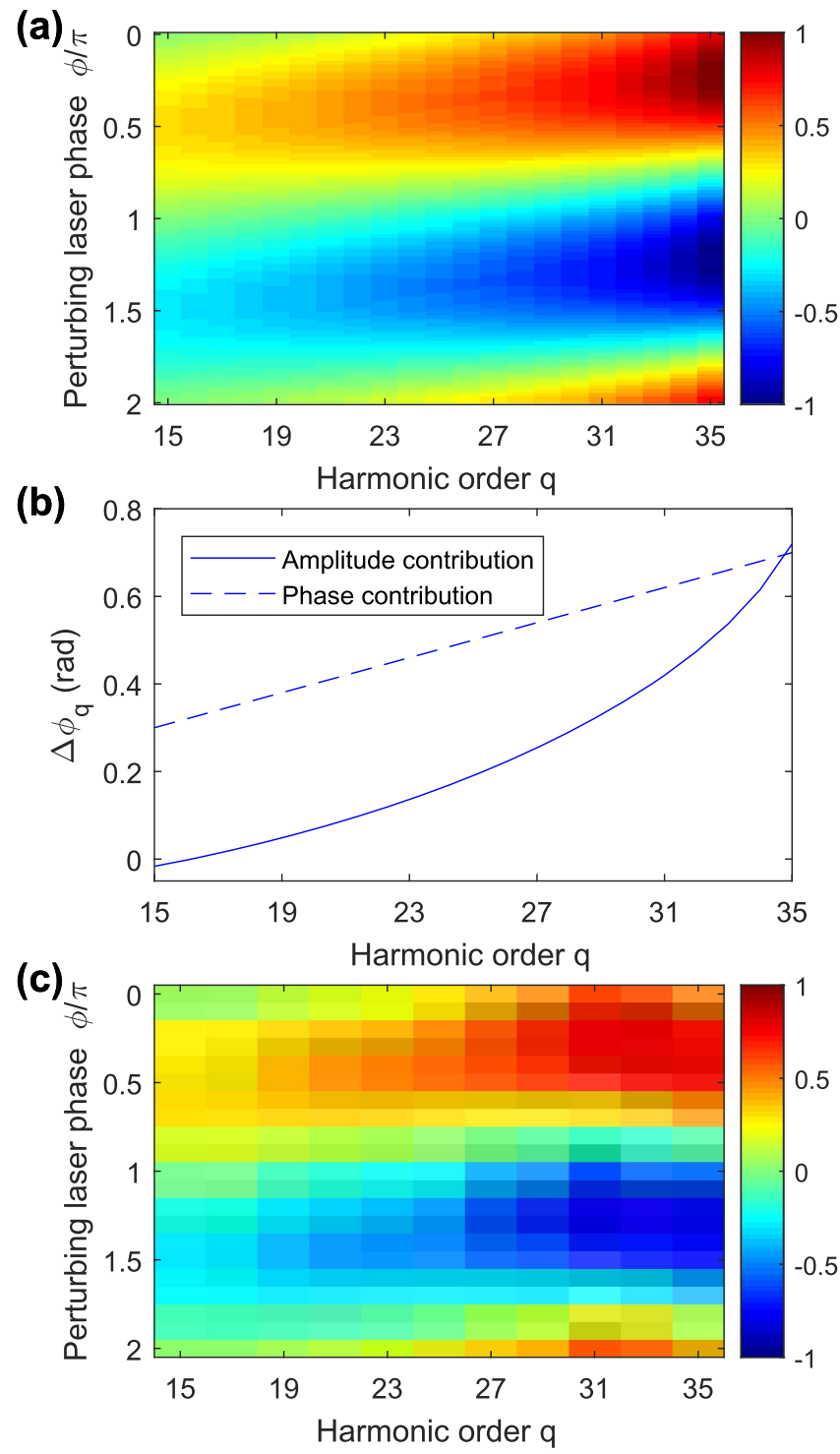

Figure 1. Theoretical analysis of high harmonic phase modulation. (a) Saddle point calculation of the phase shift (color) as a function of the harmonic order $q$ and the perturbing laser phase $\phi$. The relative perturbing laser amplitude $\delta E / E=0.02$. (b) The high harmonic phase modulations contributed by the total driving field amplitude variation (solid line) and phase variation (dashed line). (c) Timedependent Schrödinger equation (TDSE) calculation of the harmonic phase shift with $\delta E / E=0.02$.

circular high harmonic beams [29], selecting a specific high harmonic order [30], in situ focusing high harmonics like a Fresnel zone plate [31], and actively tuning the optical angular momentum of high harmonic vortices [32, 33].

We analyze a technology for an SLM analog that is applicable to high harmonic generation. A weak perturbing laser field is coherently added to the driving laser field, forming a total driving field with an amplitude change and a phase shift that can be controlled by the weak field. These amplitude and phase variations modify the electron ionization and recombination processes and thus introduce the high harmonic phase modulations. If the perturbing field is counter propagating against the driving field, high harmonic generation is enhanced via quasi-phase-matching [34]. In this paper, we first study the relation between the high harmonic phase modulation and the perturbing laser field which we label by its relative amplitude and phase to the driving field. In section 2, we analyze the perturbing laser dependent harmonic phase based on the Lewenstein strong field approximation (SFA) model [35] and verify the results with three-dimensional time-dependent Schrodinger equation (TDSE) simulations. In section 3, we experimentally determine the harmonic phase modulation in the presence of the perturbing field with different amplitudes or phases using the in situ wave front measurement technique [36]. The agreement between the theoretical and experimental results confirm that it is possible to control the spatial phase distribution of a beam by applying a perturbing laser field that is spatially structured by a conventional SLM.

\section{Theoretical analysis of high harmonic wave front modulations}

High harmonic generation can be understood through three steps: tunnel ionization of bounded electrons, laser acceleration of the freed electron wave packet, and electron recollision with its parent ion [37]. The saddle point analysis based on the SFA explicitly reveals the high harmonic phase contribution of each step [35]. If the process is perturbed by $E_{\mathrm{p}}=\delta E \cos \left(\omega_{\mathrm{p}} t+\phi\right)$ added to the intense driving field $E_{\mathrm{d}}=E \cos \left(\omega_{\mathrm{d}} t\right)$, the electron dynamics in all these three steps and the high harmonic phase are modified. Here $\delta E$ and $E$ are field amplitudes, and $\omega_{\mathrm{p}}$ and $\omega_{\mathrm{d}}$ are frequencies of the perturbing and driving beams respectively. The harmonic phase modulation due to the perturbing laser field is dominantly introduced in the free electron acceleration step [38]. Thus, the high harmonic phase modulation can be expressed as

$$
\Delta \phi_{q}=\int_{t_{\mathrm{b}}}^{t_{\mathrm{r}}}\left[k+A_{\mathrm{d}}(\tau)\right] A_{\mathrm{p}}(\tau) \mathrm{d} \tau,
$$

where $t_{\mathrm{b}}, t_{\mathrm{r}}, k$ are the birth time, the recombination time, and the canonical momentum of a specific electron wave packet trajectory corresponding to harmonic order $q$, and $A_{\mathrm{d}}, A_{\mathrm{p}}$ are vector potentials for the driving and perturbing fields respectively. For each harmonic order, $t_{\mathrm{b}}, t_{\mathrm{r}}, k$ are obtained from the saddle point calculation without considering the perturbing field.

If $\omega_{\mathrm{p}}=\omega_{\mathrm{d}}$, the total laser field retains its cosine function form. The total laser amplitude is $\left(E^{2}+\delta E^{2}+2 E \delta E \cos \phi\right)^{1 / 2}$. Its variation as a function of $\phi$ shifts the parameters $t_{\mathrm{b}}, t_{\mathrm{r}}$, and $k$, and modifies the specific electron wave packet trajectory corresponding to a harmonic order. The laser amplitude variation induced high harmonic phase modulation has to be carefully calculated with equation (1). On the other hand, the total field phase is $\arctan [\delta E \sin \phi /(E+\delta E \cos \phi)] \approx(\delta E / E) \sin \phi$, which has a $\pi / 2$ phase shift relative to the amplitude variation (we assume that $\delta E / E \ll 1$ ). The phase variation of the total driving field leads to the same shift of the birth and recombination times $t_{\mathrm{b}}$ and $t_{\mathrm{r}}$, but without changing the canonical momentum $k$. Thus, the harmonic phase modulation due to the shift 
of the time frame can be simply estimated as $q$ arctan $[\delta E \sin \phi /(E+\delta E \cos \phi)]$, whose maximum is $q \arcsin$ $(\delta E / E)$

The harmonic phase modulation as a function of perturbing laser phase $\phi$ is calculated based on equation (1) for electron wave packet short trajectories corresponding to different harmonic orders $q$, as shown in figure 1(a) for $\delta E /$ $E=0.02$. For a given harmonic order $q$, the relation between the harmonic phase shift $\Delta \phi_{q}$ and the perturbing laser phase $\phi$ is expressed by a cosine function

$$
\Delta \phi_{q}=C_{q} \cos \left(\phi-\psi_{q}\right)
$$

The $C_{q}$ parameter encodes the modulation depth of the harmonic phase, and $\psi_{q}$ encodes the delay of the harmonic phase modulation relative to the perturbing laser field. For low harmonic orders (e.g. for the parameters of our experiment, $q<25), \psi_{q} \approx \pi / 2$ and the harmonic phase modulation is in phase with the total driving field phase variation. This result implies that, although the electron wave packet trajectories for low harmonic orders are modified by the perturbing field, the modifications are negligible compared to the phase modulation because of the total driving field phase variation. For harmonic orders close to the cut-off frequency (e.g. $q=33$ ), $\psi_{q} \approx 0.24 \pi$ and the harmonic phase tends to synchronize with the total driving field amplitude variation. So electron propagation phase induced by the amplitude variation of the total driving field becomes important for harmonic orders near the cut-off. The actual delay $\psi_{q}$ between the harmonic phase modulation and the perturbing laser phase is determined by the competition between the amplitude and phase variations of the total driving field, and falls into the range between 0 and $\pi / 2$.

For the case of $\omega_{\mathrm{p}}=\omega_{\mathrm{d}}$, it is simple to separate the harmonic phase contribution due to variations in the driving field amplitude and phase. The contribution from amplitude variation can be calculated by setting $\phi=0$ for the perturbing field in equation (1) (figure 1(b), solid line). The total field phase variation induced harmonic phase modulation equals $q \arcsin (\delta E / E)$, and is plotted by the dashed line in figure 1 (b). For low harmonics (e.g. $q=15$ or 17) the harmonic phase shift is close to zero and significantly smaller than that contributed by the driving field phase variation. However, the contribution from amplitude variations increases rapidly with the harmonic order, and the two parts becomes comparable for harmonics close to the cut-off.

The above calculation for harmonic phase modulation is based on the saddle point analysis, and only takes the phase modulation contribution in the second electron acceleration step into account. We have also performed the TDSE simulation for the high harmonic phase modulation. In the simulation, a single active electron in a Coulomb potential $V(r)=-Z^{2} / r$ is driven by linearly polarized driving and perturbing fields through an interaction Hamiltonian $H_{i}=\left(E_{\mathrm{d}}+E_{\mathrm{p}}\right) r \cos \theta$ in the length gauge. Here the effective nuclear charge $Z$ is 1.077 so the ionization potential is $I_{P}=15.7 \mathrm{eV}$ (the ionization potential of argon gas), the driving field intensity is $1.5 \times 10^{14} \mathrm{~W} \mathrm{~cm}^{-2}$, the wavelength is $800 \mathrm{~nm}, r$ and $\theta$ are radial and polar variables respectively in the spherical coordinate defined by the field polarization as the zenith direction. The three-dimensional electron wave function is expanded to a series of partial waves labeled by their angular momentum $l$. The maximum angular momentum of the electron partial waves is $l_{\max }=70$, which guaranteed the convergence of the calculation. In order to exclude the long trajectory contribution to the harmonic spectrum, we set an absorbing boundary condition at $r_{\max }=30 \mathrm{a}$.u. for the wave function propagation, and a wavelet time-frequency analysis show that the long trajectory contribution is successfully suppressed [39]. The radial step is 0.1 a.u. and the time step is 0.09 a.u.. In each time step, the electron wave function propagation is calculated using the Crank-Nicolson method [40].

Figure 1(c) shows harmonic phase modulations at different perturbing field phase $\phi$. The results are consistent with the saddle point calculation except near the cut-off region because of the small radial range $r_{\max }$ and the absorbing boundary condition. Thus, we see that the ionization and recombination steps have negligible effect on the harmonic phase modulation compared to the electron propagation step. These results also suggest that the harmonic phase modulation scheme is nonlinear medium independent.

If the perturbing field has a different wavelength from the driving field, the relative phase difference $\phi$ between the driving and the perturbing fields are different for each attosecond pulse in the high harmonic radiation pulse train. Equation (1) has to be applied to calculate each attosecond pulse separately. The general definition of the harmonic phase modulation in an attosecond pulse train depends on the harmonic orders. For example, in the case of $\omega_{\mathrm{p}}=2 \omega_{\mathrm{d}}$, the neighboring two attosecond pulses are generated by the driving field with opposite directions, but are modulated by the perturbing field with the same phase. Thus, one attosecond pulse has a spectral phase shift $\Delta \phi_{q}$, whereas the other, with the opposite field direction and a half driving field cycle time delay, has a phase shift of $-\Delta \phi_{q}$. The total harmonic field at frequency $q \omega_{\mathrm{d}}$ is $E\left(q \omega_{\mathrm{d}}\right) \mathrm{e}^{\mathrm{i} \Delta \phi_{q}}-E\left(q \omega_{\mathrm{d}}\right) \mathrm{e}^{-\mathrm{i} \Delta \omega_{q}} \mathrm{e}^{-\mathrm{i} q \omega_{\mathrm{d}} T / 2}$, where $T=2 \pi / \omega_{\mathrm{d}}$. If $q$ is odd, the field is $E\left(q \omega_{\mathrm{d}}\right) \cos \Delta \phi_{q} \approx$ $E\left(q \omega_{\mathrm{d}}\right)\left[1+O\left(\Delta \phi_{q}^{2}\right)\right]$; if $q$ is even, it is proportional to $E\left(q \omega_{\mathrm{d}}\right) \sin \Delta \phi_{q} \approx E\left(q \omega_{\mathrm{d}}\right) \Delta \phi_{q}$. Thus, in the double frequency perturbation case, the high harmonic radiation is modulated in amplitude rather than in phase. The odd harmonic orders are less sensitive to the perturbing field than the even orders, though its intensity is higher. It is possible to take advantage of the difference of such sensitivity to achieve quasi-phase-matching of only even order harmonics [41].

We will concentrate on the case of $\omega_{\mathrm{p}}=\omega_{\mathrm{d}}$ in the following part of this paper. There are two reasons: (1) the conclusion drawn from the $\omega_{\mathrm{p}}=\omega_{\mathrm{d}}$ case is applicable to the phase modulation of each isolated attosecond pulse in the more complicated two-color perturbing case, and (2) for the high harmonic SLM which corrects the wave front aberration, the phase modulation in the $\omega_{\mathrm{p}}=\omega_{\mathrm{d}}$ case is more efficient than the amplitude modulation in the $\omega_{\mathrm{p}}=2 \omega_{\mathrm{d}}$ case. 


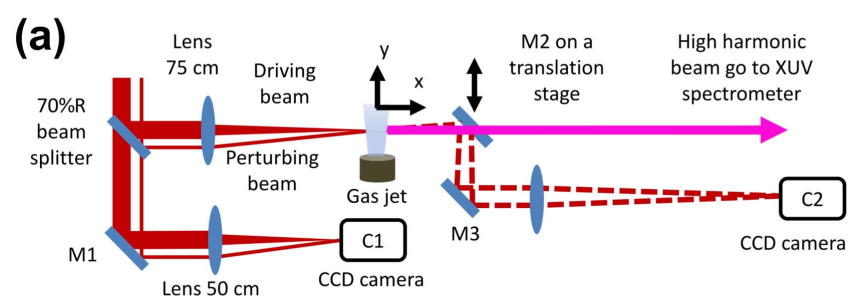

(b)

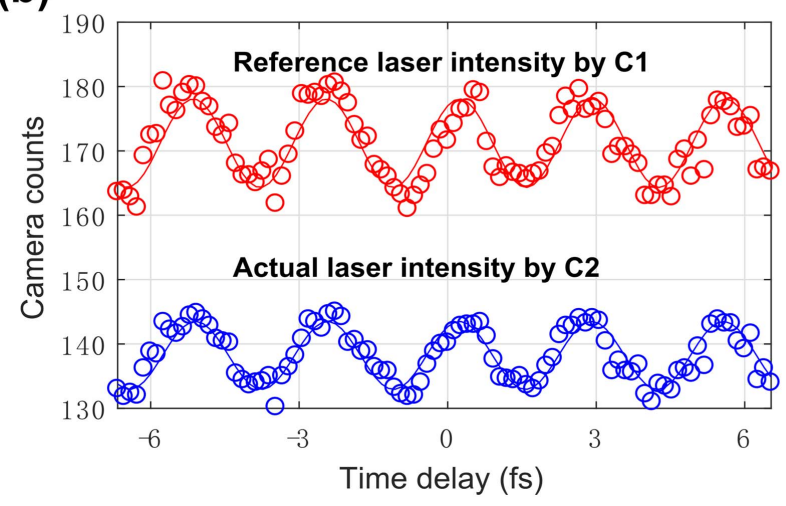

Figure 2. (a) Schematic diagram of the experimental setup. The temporal delay of the driving laser beam (thick red lines) is controlled by a piezo-stage relative to the perturbing laser beam (thin red lines). M1-3 are uncoated silica plates providing $4 \%$ reflection for the laser beams at $45^{\circ}$ incident angle. M2 is on a motorized stage. Used for calibration, it can be moved out of the high harmonic beam path in actual experiments. (b) The camera counts captured by the reference camera $\mathrm{C} 1$ (red) and the calibration camera $\mathrm{C} 2$ (blue) respectively. Measured data are labeled with circles, and the fitted sine functions are plotted with solid lines.

\section{Experimental results and discussions}

To complement our analysis, we measured the harmonic phase using the in situ method [36], while the perturbing laser field relative amplitude $\delta E / E$ and phase $\phi$ are monitored simultaneously. As shown in figure 2(a), the perturbing laser beam (wavelength $\lambda_{\mathrm{p}}=2 \pi c / \omega_{\mathrm{p}}=800 \mathrm{~nm}$ ) crossed the intense driving beam (wavelength $\lambda_{\mathrm{d}}=\lambda_{\mathrm{p}}=800 \mathrm{~nm}$, pulse duration $40 \mathrm{fs}$ ) at the gas jet at an angle of $\alpha=15 \mathrm{mrad}$. The oblique angle geometry introduced a linear perturbing laser phase shift $\phi(y)=-2 \pi y \alpha / \lambda_{\mathrm{p}}+\phi_{0}$ between the driving and perturbing field along $y$, where the first term is due to the oblique angle geometry and the second term $\phi_{0}$ is determined by the temporal delay between these beams. We tune $\phi_{0}$ with a piezo-controlled translation stage on the driving beam arm. Thus, the $q$ th harmonic wave front has a cosine function shaped phase modulation $\Delta \phi_{q}(y)=C_{q} \cos \left[\phi(y)-\psi_{q}\right]$ along the $y$-direction and at this small angle it changes the high harmonic beam pointing and the far field divergence. By scanning $\phi_{0}$, the oscillation of the harmonic beam pointing and divergence revealed the harmonic phase modulation (figure 3(a)), similar to the petahertz optical oscilloscope [42].

The perturbing laser field relative amplitude $\delta E / E$ and phase $\phi_{0}$ are measured with the assistance of a replicated reference beam pair. In figure $2(a)$, a $70 \%$-reflective beam splitter transmitted a copy (30\% of the total energy) of the harmonic generating-perturbing pulse pair and the camera $\mathrm{C} 1$

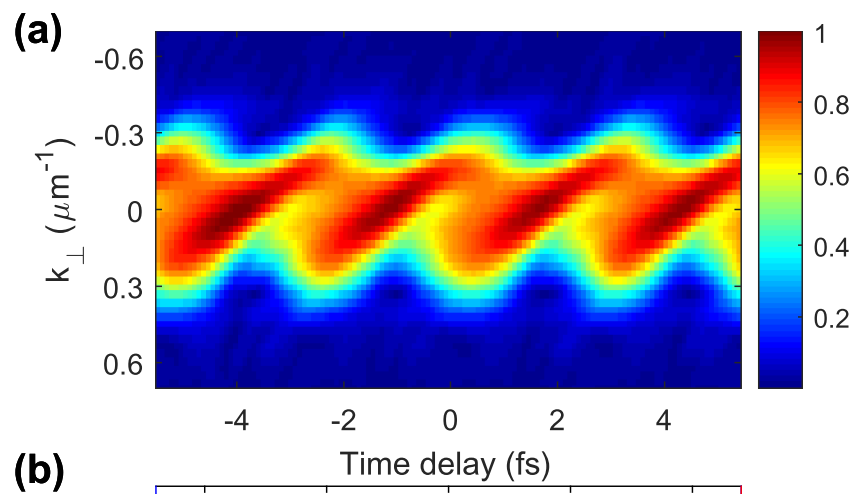

(b)
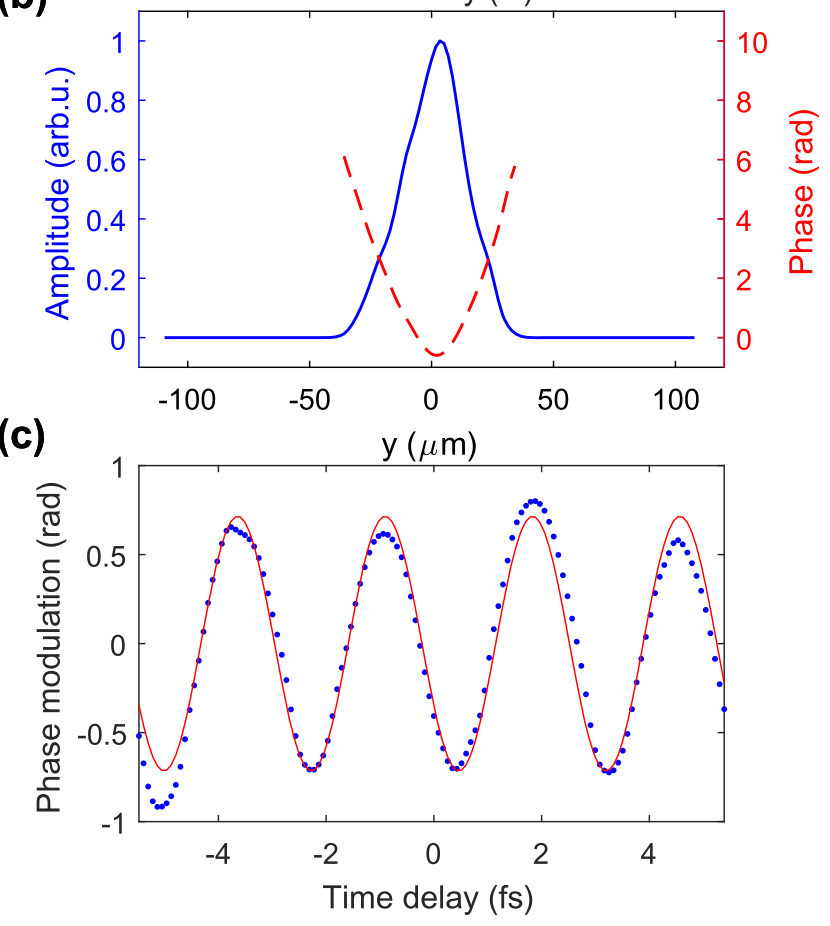

Figure 3. Reconstruction of the high harmonic phase modulation for H19. (a) The measured far field spectrogram with different temporal delay between the driving and perturbing fields. The far field diverging angle $\alpha_{q}$ is converted to the transverse wave vector $k_{\perp}$ of the harmonic beam, by $k_{\perp}=\left(2 \pi q / \lambda_{\mathrm{d}}\right) \alpha_{q}$ where $q=19$. (b) The reconstructed near field electrical profile of the H19 beam. The solid line for amplitude and the dashed line for phase. (c) The

reconstructed phase modulation of the H19 beam at $y=0$ with different temporal delay between the driving and perturbing fields (blue dotted line) and its sine function fitting (red solid line).

captured the interference of the copy at the focal spot. Experimentally mirror M2 is off-set from the XUV beam line, and the high harmonic beam is monitored with an XUV spectrometer. Thus the frequency-resolved far field profiles of the high harmonic beam and interference of the reference laser beam pair on the camera $\mathrm{C} 1$ are recorded simultaneously. In order to determine the actual perturbing laser phase and relative amplitude from the camera $\mathrm{C} 1$ interference data, a separate calibration procedure involves moving in the steering mirror M2 in the high harmonic generation chamber, using camera $\mathrm{C} 2$ to record the interference between the fundamental and perturbing beams, and comparing the pattern with the reference beam pair interference data from the camera $\mathrm{C} 1$. 
Figure 2(b) shows the $\mathrm{C} 1$ and $\mathrm{C} 2$ camera counts for the centers of the copied reference and the real focal spot at the gas target. We obtain $\phi_{0}$ by fitting the intensity signal to a cosine function. There are mainly two sources of systematic uncertainties in determining $\phi_{0}$. One is the thermal or nonlinear Kerr phase difference caused by the optics. We minimize this contribution by using a $4 \%$ reflection surface from a silica plate for mirrors M1, M2, and M3, as shown in figure 2(a). The other uncertainty is the depth of field of the imaging system from the gas target to the camera $\mathrm{C} 2$. We minimize this source by mounting a knife-edge as a reference object at the gas nozzle, and optimizing the position of the camera $\mathrm{C} 2$ by overlapping the images of the reference object illuminated by the driving and the perturbing beams respectively. Experimentally we observe visible degradation of the image on the camera $\mathrm{C} 2$ if the gas jet is moved $0.5 \mathrm{~mm}$ longitudinally along the $x$-direction. It corresponds to $\sim 0.06 \pi$ uncertainty of $\phi_{0}$. The relative field amplitude $\delta E / E$ equals $I_{\mathrm{AC}} /\left(I_{\mathrm{DC}}+\sqrt{I_{\mathrm{DC}}^{2}+I_{\mathrm{AC}}^{2}}\right)$, where $I_{\mathrm{AC}}$ the amplitude of the AC oscillation component and $I_{\mathrm{DC}}$ the averaged camera count signal, or the DC component.

To determine the high harmonic phase, we use the following algorithm to reconstruct the high harmonic phase modulation at the gas position from the far field high harmonic spectrogram measured by the XUV spectrometer (figure 3(a) for H19). Consider that the $q$ th harmonic is modulated by a function $g_{q}(y)=a_{q}(y) \mathrm{e}^{\mathrm{i} \Delta \phi_{q}(y)}$ as it is created allowing both amplitude modulation $a_{q}(y)$ and phase modulation $\Delta \phi_{q}(y)$. The measured far field high harmonic spectrogram is

$$
S_{q}\left(k_{\perp}, \phi_{0}\right)=\left|\int E_{q}(y) g_{q}\left(y-\lambda_{\mathrm{p}} \phi_{0} / 2 \pi \alpha\right) \mathrm{e}^{\mathrm{i} k_{\perp} y} \mathrm{~d} y\right|^{2},
$$

where $E_{q}(y)$ is the $q$ th harmonic near field complex profile without the perturbing field, $k_{\perp}$ the $q$ th harmonic transverse wave vector related to the far field diverging angle. Equation (3) is similar to the signal measured in the wellknown frequency-resolved optical gating technique [43], so the same principle component general projection algorithm (PCGPA) can be used to reconstruct the unperturbed harmonic field $E_{q}(y)$ and the modulation function $g_{q}(y)$.

As shown in figure 3(b), the unperturbed near field profile of H19 is reconstructed, including the amplitude (blue solid line) and phase (red dashed line) distribution along $y$ direction. The parabolic phase profile indicates a diverging wave front of the $\mathrm{H} 19$ beam at the gas jet. The measured phase modulation of the $\mathrm{H} 19$ beam $\Delta \phi_{q=19}$ at the beam center $(y=0)$ is shown by the dotted line in figure 3(c), as a function of the temporal delay between the driving and perturbing fields, effectively $\phi$. By fitting the measured data with a cosine function and comparing it with the fitted laser phase (figure 2(b)), we obtain the harmonic phase modulation depth $C_{q}$ and its delay $\psi_{q}$ relative to the perturbing laser field $\phi$.

We first consider the relative delay $\psi_{q}$ between the harmonic phase modulation and the perturbing laser field, as shown in figure 4(a). For low harmonic orders $(q \leqslant 25)$, the relative delay $\psi_{q}$ is around $(0.50 \pm 0.13) \pi$, consistent with the
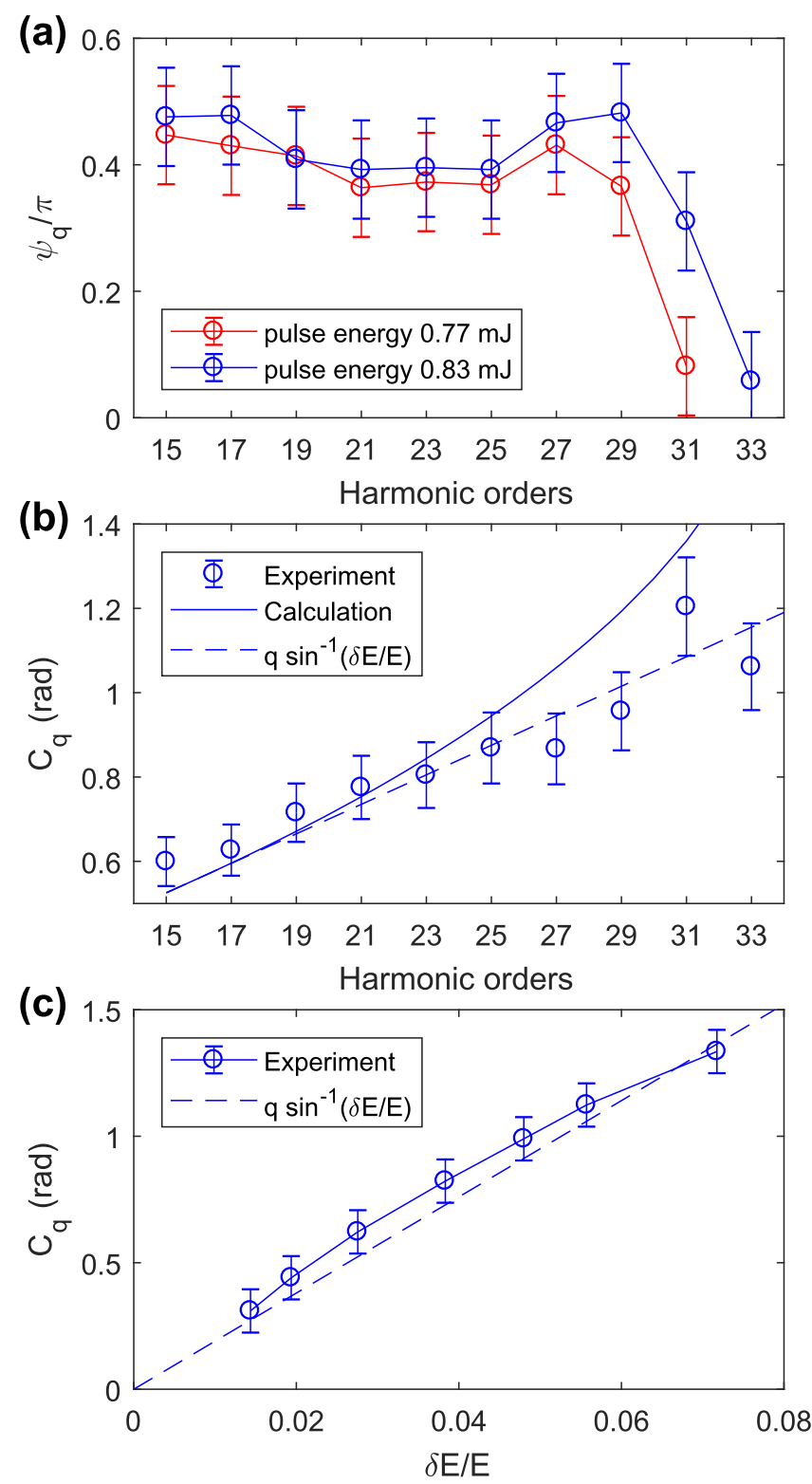

Figure 4. High harmonic phase modulation delay $\psi_{q}$ and depth $C_{q}$ in argon. (a) The harmonic modulation delay $\psi_{q}$ as a function of the harmonic order $q$. The driving laser pulse energy is $0.83 \mathrm{~mJ}$ (blue) and $0.77 \mathrm{~mJ}$ (red). (b) The harmonic modulation depth $C_{q}$ as a function of the harmonic order $q$. The experimental results are shown in circles, the saddle point calculation results in the solid line, and the dash line shows the function $q \arcsin (\delta E / E)$. In (a) and (b), the relative perturbing field amplitude is $\delta E / E=0.035$. (c) The harmonic modulation depth $C_{q}$ as a function of the relative perturbing laser amplitude $\delta E / E$ for harmonic H19. The circles and solid line show the experimental result, and the dashed line shows an estimate of the modulation depth based on the function $q \arcsin (\delta E / E)$.

theoretical analysis above. It means that the high harmonic phase modulation for low orders is predominantly determined by the phase variation of the total driving laser field. For harmonic orders close to the cut-off ( $q=33$ for $0.83 \mathrm{~mJ}$ pulse energy in blue and $q=31$ for $0.77 \mathrm{~mJ}$ in red), the parameter $\psi_{q}$ decreases to $\sim 0.1 \pi$. As discussed above, it is because the electron propagation phase modulation, which is related to the driving laser amplitude variation, becomes important compared to the phase 
variation part. For harmonic orders around $q=27$ or 29 , an increase of $\psi_{q}$ is observed that was not found in the previous theoretical analysis. This observation arises because it is experimentally difficult to suppress the long trajectory contributions near the cut-off. However, at the cut-off, the long and short trajectories merge and the analysis based on the short trajectory assumption becomes available again. In addition, for both 0.83 and $0.77 \mathrm{~mJ}$ driving laser pulse energy, the dependence of $\psi_{q}$ on the harmonic order $q$ behaves the same, implying that the relative importance between the driving field amplitude and phase variation only depend on whether the harmonic order is close to the cut-off or not.

The dependence of $\psi_{q}$ on the high harmonic orders raised the question of the feasible range of the petahertz optical oscilloscope technique [42]. In this technique, the high harmonic modulation is reconstructed either by the far field beam pointing of the high harmonic spectrogram [42], or by the PCGPA algorithm [44]. When one is interested in the perturbing laser field, especially its absolute carrier envelope phase, the equivalence between the harmonic modulation and the perturbing field depends on the harmonic orders, or photon energy for a continuous spectrum of an attosecond pulse. The existence of $\psi_{q}$ and its dependence on harmonic order $q$ suggested that a petahertz optical oscilloscope is accurate when the harmonic order is low. It is because the harmonic phase modulations for low orders are dominated by the laser field phase variation.

The harmonic phase modulation depth $C_{q}$ depends on harmonic orders as well, as calculated in the theoretical analysis. As shown in figure 4(b), the measured modulation depth $C_{q}$ increases with the harmonic order for a given perturbing field $(\delta E / E=0.035)$. The saddle point calculation of the modulation depth $C_{q}$ with the same $\delta E / E$ is shown in figure 4(b) (solid line) for comparison. For low harmonic orders, the harmonic phase shift is mainly because of the phase shift of the total driving field, so we can also evaluate the modulation depth as $C_{q}=q \arcsin (\delta E / E)$, which is linearly proportional to the harmonic order and plotted by the dashed line in figure 4(b). The laser phase variation induced modulation depth calculation starts to deviate from the saddle point calculation for harmonic orders $q \geqslant 25$, indicating that the electron propagation phase is playing a more important role for cut-off harmonics. Both the theory predictions and the experimental results showed a modulation depth in the range from 0.6 to $1.2 \mathrm{rad}$ with $\delta E / E=0.035$, a reasonable working range for an SLM.

The harmonic phase modulation depth $C_{q}$ also increases as the relative perturbing field $\delta E / E$ increases. According to equation (1), the phase modulation should be proportional to the amplitude of the perturbing field. If the perturbing field significantly increases, the total driving field amplitude variation is large enough to modify the ionization rate and the amplitude of the high harmonics. In that case, the linear dependence of $C_{q}$ on $\delta E$ in equation (1) is insufficient to describe the wave front modulation. As shown in figure 4(c), the slope of the modulation depth $C_{q}$ as a function of the relative perturbing field $\delta E / E$ drops, as the perturbing field
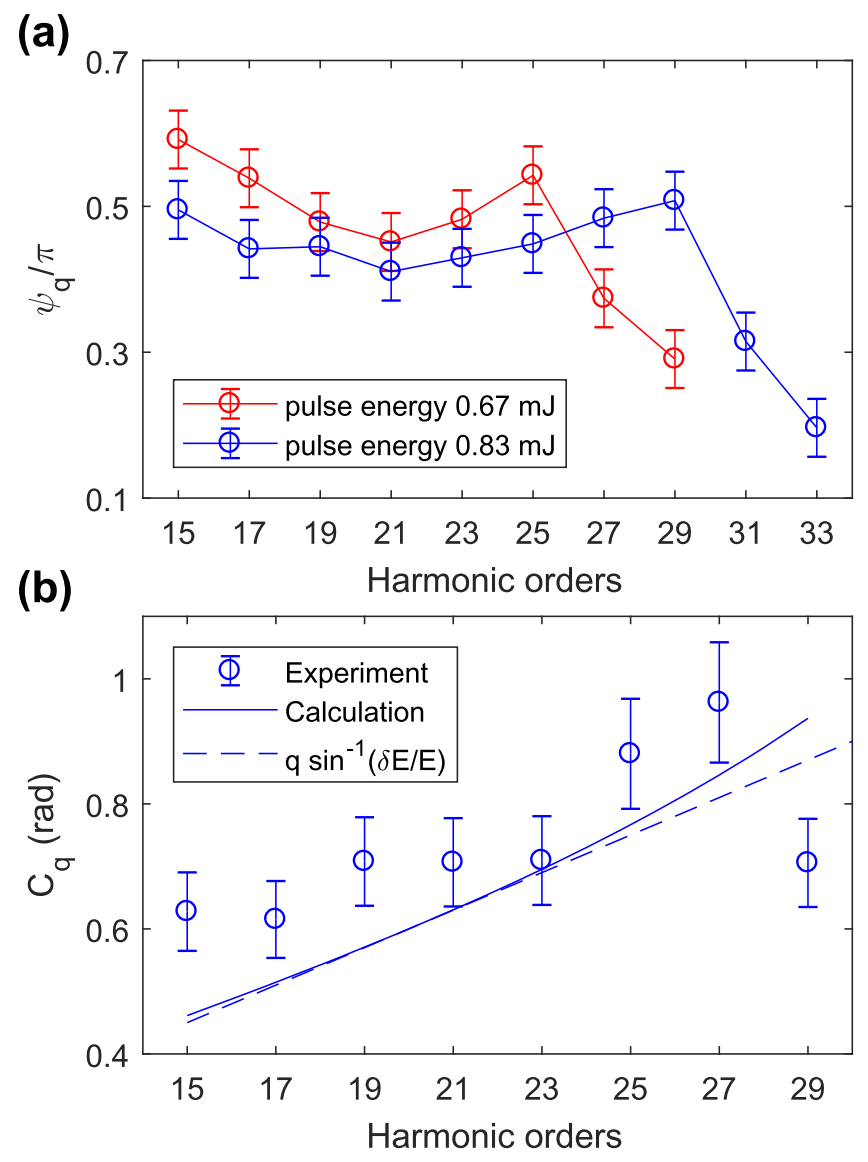

Figure 5. High harmonic phase modulation delay $\psi_{q}$ and depth $C_{q}$ in neon. (a) The harmonic modulation delay $\psi_{q}$ as a function of the harmonic order $q$. The driving laser pulse energy is $0.83 \mathrm{~mJ}$ (blue) and $0.67 \mathrm{~mJ}$ (red). (b) The harmonic modulation depth $C_{q}$ as a function of the harmonic order $q$. The experimental result is shown in circles, the saddle point calculation result in the solid line, and the dash line shows the function $q \arcsin (\delta E / E)$. The relative perturbing field amplitude is $\delta E / E=0.030$.

increases. This behavior of the modulation depth $C_{q}$ indicates a transition of high harmonic modulation from a perturbative regime to a non-perturbative regime. In addition, the amplitude modulation of the high harmonic wave front can also be obtained from the reconstruction of the harmonic near field. As the relative perturbing field $\delta E / E$ increases from 0.01 to 0.08 , the amplitude modulation increases from $5 \%$ to $40 \%$. It also explains the slow growth rate of $C_{q}$ with $\delta E / E$.

Finally, we have studied the dependence of the high harmonic phase modulation on the nonlinear gas medium, by changing it from argon to neon. As shown in the theoretical analysis, the ionization phase and the recombination phase contributions to the harmonic phase modulation is negligible compared to the electron propagation phase, thus the experimental results with neon about the harmonic phase modulation depth $C_{q}$ and delay $\psi_{q}$ in figure 5 are similar to those results with argon in figures 4(a) and (b). As shown in figure 5(a), low harmonic orders away from the cut-off generated in neon follow a $\pi / 2$ delay relative to the perturbing field, whereas the delay for higher order harmonics drops as they approach the cut-off. In figure 5(b), the measured 
modulation depth $C_{q}$ (circles) is also close to the saddle point calculation with $I_{\mathrm{p}}=21.56 \mathrm{eV}$ for neon (solid line). Note that the driving field phase variation induced modulation depth $q \arcsin (\delta E / E)$ can also reasonably predict the measured harmonic phase modulation depth, and it is independent of the gas type.

\section{Conclusion}

In conclusion, we have studied how the phase of high harmonic radiation can be modulated by the relative amplitude and phase variations of the driving laser field. Both theoretical calculations and experiments showed that for harmonic orders far from the cut-off, the $q$ th harmonic phase modulation can be estimated by $q$ times the driving laser phase variation. This relation provides a rule of thumb for high harmonic wave front control and it also defines the feasible range of the optical petahertz oscilloscope technique to measure the absolute carrier envelope phase of a few-cycle pulse.

The link between the driving laser phase and the XUV high harmonic phase also suggests an SLM for XUV high harmonic radiation. High harmonic radiation is focused and delivered by various XUV or x-ray optics for nonlinear optics or imaging applications. However, such optics (and even the extremely nonlinear high harmonic generation process itself) introduces aberrations to the wave front, leading to imperfect spatial beam profiles. In this case, a weak perturbing laser beam can be spatially modulated by an ordinary near infrared or visible SLM, and the modulated perturbing field overlaps with an intense driving field at the nonlinear medium. Thus, the perturbing field wave front pattern can be directly imprinted onto the generated high harmonic wave front to pre-compensate the aberration [45]. Moreover, with the feedback from ex situ wave front characterizations, the in situ driving-perturbing wave front control scheme would be able to work in a close loop and to realize versatile structured wave front of high harmonic radiations.

\section{Acknowledgments}

We acknowledge important financial support from the U.S. DARPA PULSE Program, from AMRDEC (Grant No. W31P4Q1310017), and the U.S. AFOSR (Grant No. FA9550-16-1-0109); The Canadian Canada Research Chairs Program, Natural Sciences and Engineering Research Council of Canada, the Canadian Foundation for Innovation, and the Ontario Research Fund. We also acknowledge invaluable technical assistance from Tyler Clancy.

\section{ORCID iDs}

Zhengyan Li (iD https://orcid.org/0000-0001-8935-8727 TJ Hammond (iD https://orcid.org/0000-0001-8334-4332

\section{References}

[1] Bagnoud V and Zuegel J D 2004 Opt. Lett. 29 295-7

[2] Chattrapiban N, Rogers E A, Cofield D, Hill W T and Roy R 2003 Opt. Lett. 28 2183-5

[3] Ostrovsky A S, Rickenstorff-Parrao C and Arrizón V 2013 Opt. Lett. 38 534-6

[4] Schonbrun E et al 2005 Opt. Express 13 3777-86

[5] Boyer V et al 2006 Phys. Rev. A 73 031402(R)

[6] Mok F, Diep J, Liu H-K and Psaltis D 1986 Opt. Lett. 11 748-50

[7] Fukushima S and Kurokawa T 1991 Appl. Phys. Lett. 58787

[8] Hayasakia Y, Sugimoto T, Takita A and Nishida N 2005 Appl. Phys. Lett. 87031101

[9] Weiner A M 2000 Rev. Sci. Instrum. 711929

[10] Chan W L et al 2009 Appl. Phys. Lett. 94213511

[11] Corkum P and Krausz F 2007 Nat. Phys. 3 381-7

[12] Ravasio A et al 2009 Phys. Rev. Lett. 103028104

[13] Gauthier D, Guizar-Sicairos M, Ge X, Boutu W, Carre B, Fienup J R and Merdj H 2010 Phys. Rev. Lett. 105093901

[14] Sekikawa T, Kosuge A, Kanai T and Watanabe S 2004 Nature 432 605-8

[15] Lambert G et al 2008 Nat. Phys. 4 296-300

[16] Attowood D 2007 Soft X-Rays and Extreme Ultraviolet Radiation: Principles and Applications (Cambridge: Cambridge University Press)

[17] Valentin C et al 2003 Opt. Lett. 28 1049-51

[18] Mashiko H et al 2004 Opt. Lett. 29 1927-9

[19] Mashiko H, Suda A and Midorikawa K 2006 Appl. Opt. 45 573-7

[20] Poletto L, Frassetto F, Calegari F, Anumula S, Trabattoni A and Nisolim M 2013 Opt. Express 21 13040-51

[21] Schnürer M et al 2000 Appl. Phys. B 70 S227-32

[22] Gautier J et al 2008 Eur. Phys. J. D 48 459-63

[23] Valentin C et al 2008 J. Opt. Soc. Am. B 25 B161-6

[24] Lambert G et al 2010 Eur. Phys. Lett. 8924001

[25] Zurch M, Kern C, Hansinger P, Dreischuh A and Spielmann C 2012 Nat. Phys. 8 743-6

[26] Gariepy G et al 2014 Phys. Rev. Lett. 113153901

[27] Rego L et al 2016 Phys. Rev. Lett. 117163202

[28] Hernández-García C et al 2017 Optica 4 520-6

[29] Hickstein D D et al 2015 Nat. Photon. 9 743C-750

[30] Rajeev R et al 2016 Light: Sci. Appl. 5 e16170

[31] Li Z et al 2017 Phys. Rev. Lett. 118033905

[32] Kong F et al 2017 Nat. Commun. 814970

[33] Gauthier D et al 2017 Nat. Commun. 814971

[34] Lytle A L et al 2008 Opt. Express 16 6544-66

[35] Lewenstein M, Balcou P, Ivanov M Y, L'Huillier A and Corkum P B 1994 Phys. Rev. A 49 2117-32

[36] Kim K T et al 2013 Nat. Phys. 9 159-63

[37] Corkum P B 1993 Phys. Rev. Lett. 71 1994-7

[38] Dudovich N et al 2006 Nat. Phys. 2 781-6

[39] Chirila C C, Dreissigacker I, van der Zwan E V and Lein M 2010 Phys. Rev. A 81033412

[40] Kulander K C, Schafer K J and Krause J L 1992 Atoms in Intense Laser Fields ed M Gavrila (New York: Academic)

[41] Diskin T and Cohen O 2014 Opt. Express 22 7145-53

[42] Kim K T et al 2013 Nat. Phot. 7 958C-962

[43] Trebino R 2000 Frequency-Resolved Optical Gating: The Measurement of Ultrashort Laser Pulses (Norwell, MA: Kluwer Academic)

[44] Kim K T, Kim K and Hammond T J 2017 J. Phys. B: At. Mol. Opt. Phys. 50024002

[45] Hammond T J, Balogh E and Kim K T 2017 J. Phys. B: At. Mol. Opt. Phys. 50014006 